\title{
Malignant Triton Tumour: Case report
}

\author{
Sandeep.V*, Rajat Kumar, Smita K,S.S. Hiremath \\ Department of Pathology, SNMedical College, Navanagar, Bagalkot, India
}

\begin{abstract}
Malignant triton tumours (MTTs) are a rare subtype of malignant peripheral nerve sheath tumour (MPNST) showing rhabdomyoblastic differentiation, which have no treatment consensus and a poor prognosis.

This case report presents case of a 71yrs/ male with swelling in Right Hand over one month. Patient had same swelling 2yrs back which was diagnosed as Peripheral Nerve Sheath Tumour on histopathology following which he underwent surgery followed by radiotherapy. Swelling reappeared at same site after 3 months.
\end{abstract}

FNAC of swelling shows large spindle cells having elongated buckled nuclei, pointed end,with varying nucleus size and coarse nuclear chromatin. Few cells are showing Rabdomyoblastic differentiation.

\section{Keywords: Malignant Peripheral Nerve Sheath Tumour, Malignant Triton Tumour, FNAC, S-100 Protein.}

\section{Introduction}

Malignant peripheral nerve sheath tumour (MPNST) accounts for about 5-10\% of all soft tissue sarcomas ${ }^{1}$. Malignant triton tumour (MTT) is a subtype of MPNST characterized by the presence of rhabdomyosarcomatous elements in a background of schwannoma cells and constitutes about $5 \%$ of all MPNSTs and follow a particularly aggressive course ${ }^{2}$. Malignant peripheral nerve sheath tumours (MPNSTs) are believed to arise from Schwann cells or nearby cells with perineural differentiation.

MTTs are identified by focal evidence of skeletal muscle differentiation within the MPNSTs. Positive immunohistochemical staining for desmin, actin and myogenin is evidence of skeletal muscle differentiation ${ }^{3}$. A recent study reported that the five-year survival rate and median survival time of patients with MTT were 14\% and 13 months, respectively ${ }^{4}$. To date, less than 100 cases of MTTs have been reported in the English literature ${ }^{4}$. Regarding the original location, MTTs occur predominantly in the head, neck and trunk regions ${ }^{5}$.

\section{Case report}

Patient 71yrs/ Male having two (2) swelling in Right Hand over last 2 month. On physical examination, the patient was found to be normal. There was a firm, nontender mass over the right hand above the wrist joint measuring $7 \times 5$ $\mathrm{cm} \& 4 \mathrm{X} 2 \mathrm{~cm}$ respectively (Fig:1). There was no palpable axillary lymphadenopathy. There was no history of trauma. He had same swelling 2yrs back for which biopsy was done and it was diagnosed as Peripheral Nerve Sheath Tumour on histopathology. He underwent surgery and radiotherapy.
Radiotherapy was given. Swelling reappeared at the same site after 3 months of radiation.

FNAC of swelling shows large spindle cells having elongated buckled nuclei, pointed end, with varying nucleus size and coarse nuclear chromatin. Few cells are showing Rabdomyoblastic differentiation. (Fig:2 \& 3).

Patient underwent surgery and resected mass was a large globular well-encapsulated tumour measuring $6 \times 5.5 \mathrm{~cm}$. Outer surface is smooth, and cut surface is solid, dull gray-white with specks of necrosis and haemorrhage. Microscopy revealed spindle cells arranged in bundles and fascicles. Individual cells are with ovoid nuclei and abundant eosinophilic cytoplasm. Atypical cells with enlarged nuclei and frequent mitosis are noted. Mitotic figures were 10-12/10 high-power fields with a few atypical mitoses (Fig:4).

Immunohistochemical stain showed malignant cells are strongly positive for S-100 protein (Fig:5).

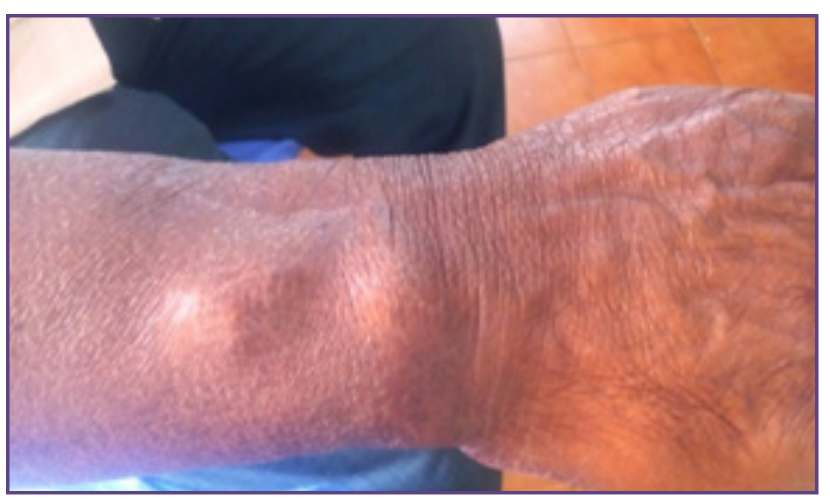

Fig. 1: Swelling in handwith previous surgery scar mark. 


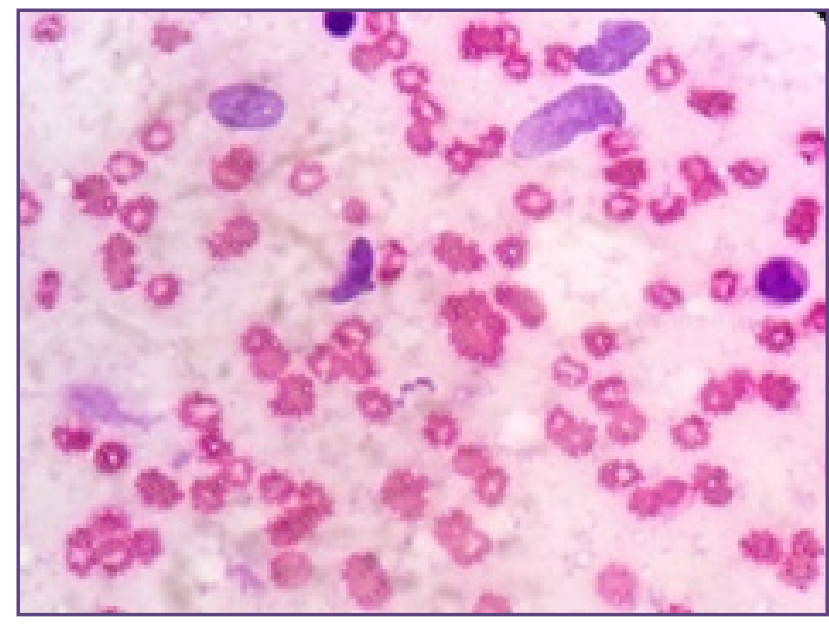

Fig. 2: Few cells are showing Rabdomyo blastic differentiation (H \& E, 40X).

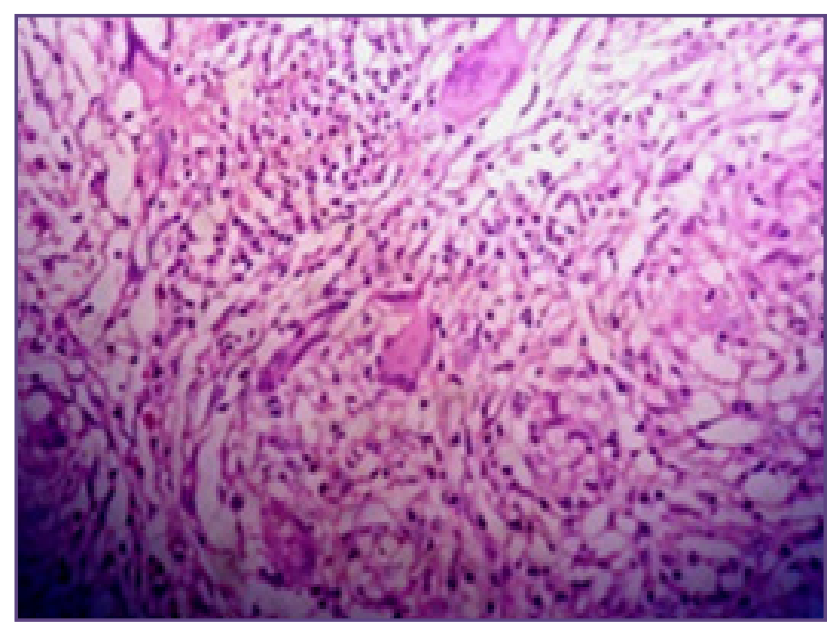

Fig. 4: Mitotic figures were 10-12/10 high-power fields with a few atypical mitoses( H\&E 10X)

\section{Discussion}

The first description of MTT dates back to 1932, when Masson described rhabdomyosarcomatous elements within MPNST ${ }^{6}$. There are 3 criteria for diagnosing MTT. (I)Tumour related to Peripheral Nerve/ NF-1. (II) Most of tumour consist of Schwann Cells. (III)Tumour contains Rhabdomyoblastic differentiation. No difference between MTT \& MPNST in terms of the rate of local recurrence $\&$ rate of Metastasis. MTTs may arise in association with NF-1 or can arise sporadically ${ }^{7}$. Two third of the cases of MTTs have been reported in conjunction with NF-1. When it occurs in the sporadic form, other spindle cell sarcomas such as fibrosarcoma, malignant fibrous histiocytoma, and rhabdomyosarcoma can come as differential diagnosis8. MTT has a poor prognosis and local recurrence has been observed to be common following tumour excision, while

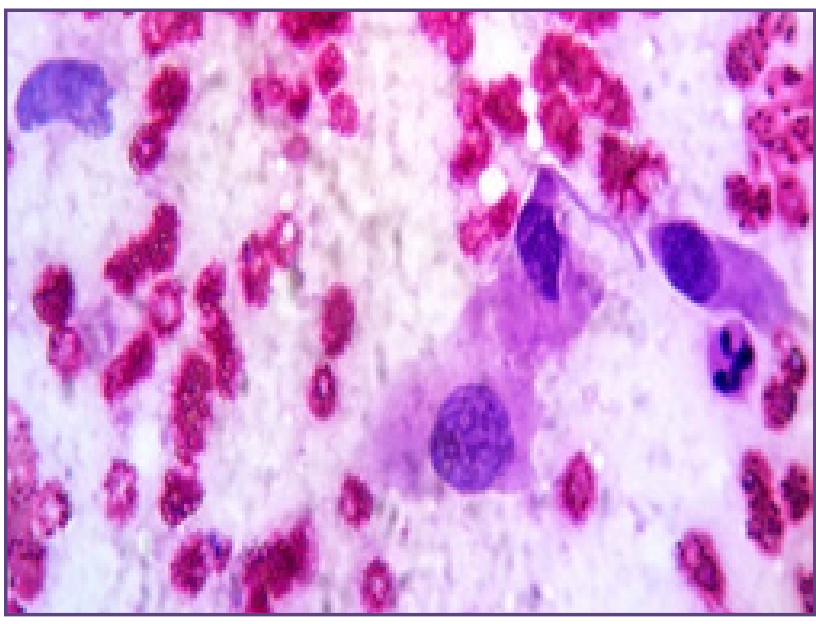

Fig. 3: Few cells are showing Rabdomyoblastic differentiation(H\&E 100X).

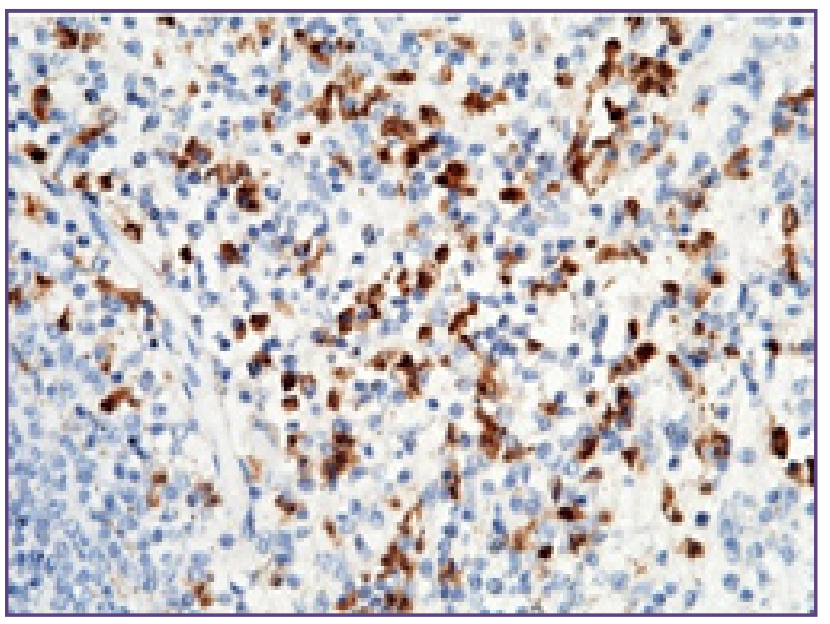

Fig. 5: S-100 positive

lymphatic invasion and lymph node involvement has not been reported in patients with $\mathrm{MTT}^{2}$.

Histological analysis revealed a spindle cell tumor with interlacing fascicles of wavy spindle cells and prominent mitotic figures. Loose and impact arrangements were detected. In certain focal areas, the tumor was comprised of round rhabdomyoblasts, with abundant eosinophilic cytoplasm and eccentric nuclei in a loose matrix. ${ }^{12}$

The diagnosis of MTT is usually made by histopathology examination \& conformed by demonstration of S-100 protein ${ }^{5,9}$. The morphologic features are alternating hypocellular and hypercellular regions with spindle cells in bundles and fascicles with ovoid nuclei and abundant eosinophilic cytoplasm and presence of rhabdomyoblasts. Such tumours show focal positivity for S-100 protein 
in 50-90\% of cases, suggesting a nerve sheath origin. Rhabdomyoblasts are positive for immunohistochemical stains such as desmin, myogenin, and myo-D1 ${ }^{10}$. Our case was positive for S 100 and myogenin. MTTs occur predominantly in the head, neck and trunk regions. In total, $\sim 20 \%$ of MTT cases arise in the head and neck, with $32 \%$ in the trunk and $24 \%$ in the extremities ${ }^{11}$. In our case, the patient had swelling in the lateral aspect of right arm and the tumour recurred from a previous MPNST.

\section{Conclusion}

MTT is a rare neurogenic tumour with rhabdomyoblastic differentiation. Histopathology with support of Immunohistochemistry remains the mainstay of investigation to establish the diagnosis. Given the aggressive nature of the tumour with poor prognosis, radical excision followed by adjuvant chemoradiotherapy appears to improve survival.

\section{References}

1. Weiss SW, Goldblum JR. Malignant tumours of peripheral nerves. In: Weiss SW, Goldblum JR (eds) Enzinger and weiss's soft tissue tumours, 5th edn. Mosby Elsevier, China, 2008: 903-944.

2. Brooks JSJ. Disorders of soft tissue. In: Sternberg SS (ed) Diagnostic surgical pathology, 3rd edn. Lipincott Williams and Wilkins, Philadelphia, 1999: 131-221.

3. Scheithauer BW: Malignant peripheral nerve sheath tumour (MPNST). In: WHO Classification of Tumours of the Central Nervous System. Louis DN (ed). World Health Organization Publ., Corp., Lyon, pp160-162, 2007.
4. McConnell YJ and Giacomantonio CA: Malignant triton tumours-complete surgical resection and adjuvant radiotherapy associated with improved survival. J Surg Oncol 2012:106: 51-56.

5. Brooks JS, Freeman M and Enterline HT: Malignant triton tumour. Natural history and immunohistochemistry of nine new cases with literature review. Cancer 55: 2543-2549, 1985.

6. Masson P: Recklinghausen's Neurofibromatosis, Sensory Neuromas and Motor Neuromas. International Press, New York, NY, 1932.

7. Leroy K, Dumas V, Martin-Garcia N et al (2001) Malignant peripheral nerve sheath tumours associated with neurofibromatosis type 1: a clinicopathologic and molecular study of 17 patients. Arch Dermatol 137:908-913.

8. Aldlyami E, Dramis A, Grimer RJ et al (2006) Malignant triton tumour of the thigh - a retrospective analysis of nine cases. Eur J Surg Oncol 32:808-810.

9. Stasik CJ, Twafik O (2006) Malignant peripheral nerve sheath with rhabdomyosarcomatous differentiation (malignant triton tumour). Arch Pathol Lab Med 130:1878-1881.

10. Daimaru Y, Hashimoto H, Enjoli M (1984) Malignant triton tumours. A Clinicopathologic and immunohistochemical study of nine cases. Hum Pathol 15:768-778.

11. Bhatt S, Graeme-Cook F, Joseph MP and Pilch BZ: Malignant triton tumour of the head and neck. Otolaryngol Head Neck Surg 105: 738-742, 1991.

12. Ren W, Xu X, Yan J, Qian X, Lie B:Malignat triton tumour of anterior mediastinum: A case report.Oncol Lett,2014,7(3),807-810.

*Corresponding author:

Dr. Sandeep (Post Graduate Student), Department of Pathology, S.N Medical College, Navanagar, Bagalkot-587102

Phone: +91 9990402447

Email: sandeepc309@gmail.com

Financial or other Competing Interests: None. 